\title{
Managing Interoperability \& Complexity in Health Systems
}

\author{
M-M. Bouamrane ${ }^{1}$; C. Tao ${ }^{2}$; I.N. Sarkar ${ }^{3}$ \\ 1. Institute of Applied Health Sciences, University of Aberdeen, Scotland, U.K. \\ 2. School of Biomedical Informatics, UT Health Science Center at Houston, U.S. \\ 3. Center for Clinical and Translational Science, College of Medicine, University of Vermont, U.S.
}

Keywords: Medical Informatics, Health Information Management, Knowledge Bases, Biological Ontologies, Systematized Nomenclature of Medicine, Information Storage and Retrieval, Classification, Systems Integration

Summary: In recent years, we have witnessed substantial progress in the use of clinical informatics systems to support clinicians during episodes of care, manage specialised domain knowledge, perform complex clinical data analysis and improve the management of health organisations' resources. However, the vision of fully integrated health information eco-systems, which provide relevant information and useful knowledge at the point-of-care, remains elusive. This journal Focus Theme reviews some of the enduring challenges of interoperability and complexity in clinical informatics systems. Furthermore, a range of approaches are proposed in order to address, harness and resolve some of the many remaining issues towards a greater integration of health information systems and extraction of useful or new knowledge from heterogeneous electronic data repositories.

\section{Introduction}

The seeds for this issue with a Focus Theme on 'Managing Interoperability \& Complexity in Health Systems' (MIXHS) were sown a few years ago in a 'meeting of minds' at the 9th International Semantic Web Conference (ISWC 2010) ${ }^{1}$ in Shanghai [1,2]. Our main motivation behind the organisation of a series of workshops dedicated specifically to the topics of interoperability and complexity in clinical informatics was the lack of dedicated forums focussing on these issues in international computer science conferences, with the exception perhaps of the (now bi-annual) International Medical Informatics Association (IMIA) World Congress on Medical and Health Informatics (Medinfo) ${ }^{2}$.

Since 2010, we have organised two very successful MIXHS workshops in conjunction with the ACM Conference on Information and Knowledge Management (CIKM) in Glasgow (Scotland) in 2011 and Maui (U.S.A.) in 2012 [3,4]. Both events turned out to be inspiring forums to share expertise and experience on a variety of topics: from the challenges involved in implementing truly interoperable electronic health 'ecosystems' in practice to the range of methods which can be used in order to

\footnotetext{
${ }^{1}$ http://iswc2010.semanticweb.org/

2 http://www.imia-medinfo.org/new2/
} 
extract knowledge and manage information from complex biomedical data sets. The main motivation of MIXHS is to build bridges across the disciplines of computer science, clinical informatics, healthcare and health services research so that a multi-disciplinary approach can instruct the design, development and implementation of current and future clinical informatics system.

This Focus Theme includes original research contributions both from participants of the MIXHS workshops as well as from the wider research community.

\section{Background \& State-of-the Art}

\subsection{Interoperability}

As others have highlighted in the past, the term 'interoperability' has many definitions and - in practice - is often characterised by its absence. In other words: one only really becomes aware of the concept of interoperability when it is lacking: i.e. when 'systems just won't work together'.

The Health Level Seven (HL7) Electronic Health Record (EHR) Interoperability Working Group recognised the challenge involved in providing a single definition for the term interoperability and identified 3 distinct types of interoperability challenges [5]:

(i) Technical Interoperability (i.e. the exchange of data between 2 systems),

(ii) Semantic Interoperability (i.e. the shared meaning of data across systems), and

(iii) Process Interoperability (i.e. the integration of systems within work processes)

Despite the increasing recognition of the importance and potential benefits of interoperability for patients and health systems alike, implementing truly interoperable Health IT 'ecosystems' remains a considerable challenge for a number of reasons. Benson suggested that obstacles to interoperability include: heterogeneous data formats across systems, clinical information complexity, knowledge specific to certain medical specialties, clinical context, over-complexity of standards, the lack of shared-meaning and misunderstandings between system suppliers and end-users [6].

In the U.K., the lack of interoperability between electronic health systems has been identified as a key barrier to the creation of a mass-market in consumer health and well-being technologies. Hence, achieving interoperability across the three levels of data, semantics and processes has been set as one of the strategic priorities of the Technology Strategy Board Delivering Assisted Living Lifestyles at Scale (Dallas) implementation programme [7].

In the U.S.A, the U.S. Department of Health and Human Services (HHS) and the Office of the National Coordinator for Health Information Technology (ONC) have also recognised the critical need to advance the connectivity and interoperability of electronic health information technology and health data [8].

In the U.K., eHealth systems interoperability is becoming all the more important as the adoption of patient electronic medical records within primary care is now wide-spread. This situation now provides opportunities to transfer and share patient electronic information across organisation within 
the National Health Service (NHS) that were previously unimaginable with paper-based records [9-13]. The next integration challenges will thus consist in enabling secure and efficient information sharing with other health organisations (e.g. secondary care), across specialties within organisations and increasingly with the patients themselves [14]. However, several studies have highlighted that the implementation of enterprise electronic medical records systems in secondary care across the NHS has been much more challenging than had been initially anticipated [15]. This perhaps in turn suggests that policymakers and technical providers have previously underestimated the complexity of the tasks and the efforts required to deploy organisation-wide electronic health records systems at scale [16, 17]. What these studies suggest is that the implementation of large scale integrated and interoperable eHealth systems is often difficult in practice, and often particularly so at the organisational or processinteroperability level. Yet, studies have also demonstrated that such implementations are indeed possible when a number of converging factors are in place; for example: policy support and incentives (e.g. performance targets), a thorough understanding of the environment in which the systems will operate (i.e. clinical pathways), adequate time and resources to allow for the development of technologically fit-for-purpose systems as well as the necessary service-redesign once new systems are operationalized, contextualised implementations - and last but not least - a sustained engagement with stakeholders throughout the iterative phases of systems design, development, testing and roll-out [18].

\subsection{Complexity}

Medicine is intrinsically a highly specialised, complex and evolving discipline and the inherent challenges involved in developing adequate clinical informatics systems fit-for-purpose at the pointof-care are often further compounded by the wide variations that exist across health services and organisations [19-21]. This means that, in practice, systems that can be effective within certain clinical specialties or organisational contexts can also be difficult to successfully transpose into other clinical settings.

Furthermore, health data typically reside within disparate data repositories and departmental systems (i.e. data silos), under different (and often weak) information models, in different syntaxes, semantics, or formats. Data are often captured in inconsistent or incompatible formats. The mechanisms to 'harvest' data for specific purposes are not formalised or readily available. EHR data are often of variable quality and contains large amount of human language narratives, which present considerable challenges for automatic processing [22]. Making sense of heterogeneous health data to produce relevant information and actionable knowledge often requires complex data operations and transformations, such as error-checking, standardisation, normalisation, alignment, and enrichment in order to produce information suitable for large-scale integration, analysis and/or inferencing. Mayo Clinic's Enterprise Data Trust is one example of a heterogeneous data integration approach that spans a complex healthcare organisation [23]. It was designed to support Mayo Clinic's analytic and decisionmaking processes: it enables the combination of patient, genomic, research and administrative data to support information access and retrieval and business intelligence.

\section{Focus Theme Overview}

The articles included in this Focus Theme address a range of the aforementioned fundamental challenges. 
De Backere et al. present a platform for switching between multiple devices in the Intensive Care Unit (ICU) [24: Journal Ref: De Backere et al.]. They anticipate that the platform could be used to switch between a range of electronic devices (e.g. smart-phones, tablets, desktop computers) to provide relevant information and decision support functionalities to the clinicians in the ICU. The platform was evaluated in terms of workload and performance and the authors found that the platform could support up to 20 devices without degradation in performance. As always, the real challenges in this type of implementations are to integrate the platform with the hospital information systems, to provide relevant and meaningful information to support clinician decision making and to embed systems within routine workflows in the context of the challenging environment of the ICU [25-26]. We therefore look forward to the authors' future work in this sphere.

In developed countries, most of the clinical encounters take place in a primary care settings and hence family doctors are often the gate-keepers to data-rich, longitudinal patient records containing a wealth of information about patients' conditions and their evolution over-time [11]. Ethier et al. present an interoperability framework called the Clinical Data Integration Model using an ontology to support data integration of heterogeneous primary care electronic repositories in order to support research activities (i.e. queries) of primary care data [27: Journal Ref: Ethier et al.].

Declerck et al. present an interoperability platform and toolkit aiming to enable secondary use of EHR data for drug-related, adverse-events surveillance [28: Journal Ref: Declerck et al.]. Terminology mapping and reasoning services are used for the automatic conversion of EHR terminologies (ICD-9CM, ICD-10, LOINC or SNOMED-CT) to the target adverse-event terminology. Their approach hence allows for the partial automation of the adverse-event reporting process to improve pharmacovigilance.

Liang et al. present a framework for the semi-automated integration and linkage of heterogeneous data sets (stroke register, London air pollution and clinical practice research data link) by generating OWL (Web Ontology Language) representations of these datasets [29: Journal Ref: Liang et al.]. The $O W L$ representation of the linked data sets can hence be used for querying and reasoning. This type of data transformation into OWL has also been used elsewhere for generating knowledge-bases from legacy health information systems in order to provide clinical decision support functionalities $[1,30]$.

Bache et al. present a clinician-readable computational notation, integrating temporal semantics, in order to query heterogeneous data sources to check whether patients meet eligibility criteria for clinical trials [31: Journal Ref: Bache et al.].

Berges et al. present results for comparing the performance of term-binding algorithms for SNOMEDCT terms and Archetype elements [32: Journal Ref: Berges et al.]. They suggest that string comparison-based methods can be useful to provide reduced set of candidate-terms in order to support and facilitate manual term-alignment.

Anguita et al. propose an approach to enhance RDF-based schema alignment techniques, by providing a coherent method to represent elements with context-dependent semantics, thus enabling users to perform more expressive alignment in RDF-based biomedical data [33: Journal Ref: Anguita et al.] 
Quesada-Martínez et al. present an approach to identify lexical patterns for semi-automatic ontology enrichment [34: Journal Ref: Quesada-Martínez.] Ontology developers can use these patterns to improve the axiomatisation of their ontologies.

Jiang et al. discuss their research on integrating clinical information models and clinical research data management system through the harmonization of the Intermountain Healthcare Clinical Element Models (CEMs) with the Clinical Data Interchange Standards Consortium (CDISC) data standards [35: Journal Ref: Jiang et al.]

\section{Acknowledgements}

We would like to express our extreme gratitude to all of those who made this special issue possible through their dedicated efforts to promote knowledge sharing in the field of clinical Informatics. First of all, we would like to thank Professor Reinold Haux (Peter L. Reichertz Institute for Medical Informatics of the University of Braunschweig - Institute of Technology and of Hannover Medical School) for his positive response to this special issue proposal. We would also like to thank Ina Hoffmann (Methods of Information in Medicine) for assistance with coordinating this special issue.

We would also like to thank all the reviewers who provided time and efforts to provide constructive feedback to the authors. All the articles were substantially improved as a result of the comments provided by the reviewers. 


\section{References}

1. Bouamrane, M.-M.; Rector, A. \& Hurrell, M. Experience of Using OWL Ontologies for Automated Inference of Routine Pre-operative Screening Tests The Semantic Web - ISWC 2010, Springer Berlin Heidelberg, 2010, 6497, 50-65

2. Tao, C.; Solbrig, H.; Sharma, D.; Wei, W.-Q.; Savova, G. \& Chute, C. Time-Oriented Question Answering from Clinical Narratives Using Semantic-Web Techniques. The Semantic Web - ISWC 2010, Springer Berlin Heidelberg, 2010, 6497, 241-256

3. Bouamrane, M.-M. \& Tao, C. Managing interoperability and compleXity in health systems - MIXHS'11 Proceedings of the 20th ACM international conference on Information and knowledge management, CIKM'11, Glasgow, Scotland, 2011, 2635-2636

4. Tao, C. \& Bouamrane, M.-M. Managing interoperability and compleXity in health systems - MIXHS'12 Proceedings of the 21st ACM international conference on Information and knowledge management, CIKM'12, Maui, Hawaii 2012, 2758-2759

5. Gibbons P et al. Coming to Terms: Scoping Interoperability in Health Care. Health Level Seven EHR Interoperability Work Group; 2007. https://www.hln.com/assets/pdf/Coming-to-Terms-February-2007.pdf

6. Benson, T. Principles of Health Interoperability HL7 and SNOMED. Health Informatics series. Springer, Berlin (2010)

7. McGee-Lennon, M; Bouamrane, M-M; Barry, S et al. Evaluating the Delivery of Assisted Living Lifestyles at Scale (dallas). Proceedings of HCI 2012 - People \& Computers XXVI, The 26th BCS Conference on Human Computer Interaction, Birmingham, UK, 12 - 14 Sept. 2012

8. Connecting Health and Care for the Nation: A 10-Year Vision to Achieve an Interoperable Health IT Infrastructure. The Office of the National Coordinator for Health Information Technology. June 2014, 13p. http://www.healthit.gov/sites/default/files/ONC10yearInteroperabilityConceptPaper.pdf

9. Bouamrane, M.-M. \& Mair, F. An overview of electronic health systems development and integration in Scotland Proceedings of Managing Interoperability and Complexity in Health Systems, MIXHS'11, 20th ACM Conference on Information and Knowledge Management, CIKM 2011, Glasgow, United Kingdom, October 2428, 2011, ACM, 2011, 59-62

10. Bouamrane, M.-M. \& Mair, F. A study of general practitioners' perspectives on electronic medical records systems in NHSScotland. BMC Medical Informatics and Decision Making, 2013, 13:58

11. Bouamrane, M.-M. \& Mair, F. A qualitative evaluation of general practitioners' views on protocol-driven eReferral in Scotland. BMC Medical Informatics and Decision Making, 2014, 14:30

12. Bouamrane, M.-M. \& Mair, F. A study of clinical and information management processes in the surgical preassessment clinic. BMC Medical Informatics and Decision Making, 2014, 14:22

13. Bouamrane, M.-M. \& Mair, F. A study of Information Management in the Patient Surgical Pathway in NHSScotland in Proceedings of the 14th World Congress on Medical and Health Informatics, Medinfo 2013, Copenhagen, Denmark, August 2013, 2013. Studies in Health Technology and Informatics. Volume 192: MEDINFO 2013, 557-61.

14. de Lusignan S, Ross P, Shifrin M, Hercigonja-Szekeres M, Seroussi B. A comparison of approaches to providing patients access to summary care records across old and new Europe: an exploration of facilitators and barriers to implementation. Studies in Health Technology and Informatics. Volume 192: MEDINFO 2013,397 $-401$

15. Sheikh, A.; Cornford, T. et al. Implementation and adoption of nationwide electronic health records in secondary care in England: final qualitative results from prospective national evaluation in "early adopter" hospitals. BMJ, 2011;343:d6054

16. Eason K, Waterson P. Fitness for purpose when there are many different purposes: Who are electronic patient records for? Health Inform J 2014, 20(3): 189-198

17. Waterson P: Health information technology and sociotechnical systems: A progress report on recent developments within the U.K. National Health Service (NHS). Appl Ergon 2014, 45(2, Part A): 150 - 161

18. Bouamrane MM and Mair F. Integrated Preoperative Care Pathway - A Study of a Regional Electronic Implementation. BMC Medical Informatics and Decision Making 2014, 14:93

19. Rector, AL. Clinical terminology: why is it so hard? Methods Inf Med. 1999 Dec;38 (4-5):239-52. 
20. Kannampallil TG, Schauer GF, Cohen T, Patel VL. Considering complexity in healthcare systems. Journal of Biomedical Informatics. 44 (2011) 943-947

21. Cases, $M$ et al. Improving data and knowledge management to better integrate health care and research. Journal of Internal Medicine. 2013, 274, 321-328

22. Strategic Health IT Advanced Research Projects (SHARP), Area 4 on secondary usage of EHRs, http://sharpn.org

23. Chute, C. G.; Beck, S. A.; Fisk, T. B. \& Mohr, D. N. The Enterprise Data Trust at Mayo Clinic: a semantically integrated warehouse of biomedical data. Journal of the American Medical Informatics Association, JAMIA, 2010, 17, 131-135

\section{4. [Journal Ref: De Backere et al.] Methods Inf Med. 2015; 54(1): This issue}

25. Goosh P, Roudsari A. Computerization of workflows, guidelines and care pathways: a review of implementation challenges for process-oriented health information systems. J Am Med Inform Assoc. 2011; 18:738-748

26. Musen MA, Middleton B, Greens RA. Clinical Decision-Support Systems. E.H Shortliffe, J.J. Cimino (eds), Biomedical Informatics, 22:643-674. Springer-Verlag London 2014

27. [Journal Ref: Ethier et al.] Methods Inf Med. 2015; 54(1): This issue

28. [Journal Ref: Declerck et al.]. Methods Inf Med. 2015; 54(1): This issue

29. [Journal Ref: Liang et al.] Methods Inf Med. 2015; 54(1): This issue

30. Bouamrane, M.M., Rector, A., Hurrell, M.: Semi-Automatic Generation of a Patient Preoperative KnowledgeBase from a Legacy Clinical Database. In: Proceedings of 8th International Conference on Ontologies, DataBases, and Applications of Semantics, ODBASE'09, On The Move to Meaningful Internet Systems Conferences, Vilamoura, Algarve, Portugal, Springer Lecture Notes in Computer Science,. Volume LNCS 5871/2009. (2009) 1224-1237

31. [Journal Ref: Bache et al.] Methods Inf Med. 2015; 54(1): This issue

32. [Journal Ref: Berges et al.] Methods Inf Med. 2015; 54(1): This issue

33. [Journal Ref: Anguita et al.] Methods Inf Med. 2015; 54(1): This issue

34. [Journal Ref: Quesada-Martínez.] Methods Inf Med. 2015; 54(1): This issue

35. [Journal Ref: Jiang et al.] Methods Inf Med. 2015; 54(1): This issue 